\title{
Formación de habilidades blandas en los estudiantes de la Universidad Católica de EI Salvador
}

\author{
Iris Yamileth Jiménez \\ Maestra en Profesionalización en la Docencia Superior \\ Docente investigadora, Facultad de Ciencias y Humanidades \\ Universidad Católica de El Salvador, El Salvador \\ Email: iris.jimenez@catolica.edu.sv \\ ORCID: https://orcid.org/0000-0003-2091-4064
}

Fecha de recepción: 29-01-2021

Fecha de aceptación: 27-02-2021

\section{Resumen}

Esta investigación tuvo como propósito principal clasificar las habilidades blandas de los estudiantes de la Universidad Católica de El Salvador. Se atendieron a tres categorías las habilidades blandas que el siglo XXI exige al profesional, se identificó los tipos de habilidades blandas que los alumnos poseen actualmente; y los tipos de habilidades blandas que se forman en los alumnos dentro del proceso de enseñanza aprendizaje.

El estudio se realizó a través de una investigación cualitativa y para la recolección de datos se aplicó la técnica de la encuesta a 343 estudiantes de primer y quinto año de las cuatro facultades, la entrevista a 24 docentes de materias humanísticas, materias comunes y de especialidad; y la observación dentro del salón de clases de los mismos docentes que participaron en la investigación.

Se encontró que las habilidades blandas que los estudiantes poseen son trabajo en equipo, comunicación, perseverancia, resolución de problemas y orientación al servicio. Mientras que los docentes manifestaron que las habilidades blandas que desarrollan en clases son la comunicación, empatía, puntualidad, solidaridad, respeto, amabilidad, trabajo en equipo y responsabilidad.

Palabras clave: Habilidades blandas, habilidades actuales, fomento de habilidades.

\begin{abstract}
The main purpose of this research was to classify the soft skills of the students of Universidad Católica de El Salvador. The soft skills that the XXI century demands from the professional were taken into three categories; the types of soft skills that students currently possess were identified; and the types of soft skills that are formed in students within the teaching-learning process.

The study was carried out through qualitative research, and for data collection, the survey technique was applied to 343 first and fifth year students from four faculties, the interview to 24 teachers of humanistic subjects, common subjects and specialty; and the observation within the classroom of the same teachers who participated in the research.

The findings show that the soft skills that students possess are teamwork, communication, perseverance, problem solving and service orientation. While teachers stated that the soft skills developed in class are communication, empathy, punctuality, solidarity, respect, kindness, teamwork and responsibility.
\end{abstract}

Key words: Soft skills, current skills, skill building. 


\section{Introducción}

En la actualidad las habilidades blandas forman parte del perfil de estudiantes de nuevo ingreso a nivel superior, tanto que en algunos países las grandes empresas solicitan trabajar de la mano con este nivel, para que estos aspirantes cumplan las expectativas que ellos demandan, ya que no solo se solicitan habilidades cognitivas (habilidades duras), en su currículo; si no también habilidades sociales (habilidades blandas). Para Rubilar (2016), "Las competencias blandas se han convertido en un lugar común a la hora de hablar de empleabilidad y desarrollo profesional. Desde hace algunos años se ha ido convirtiendo en un requerimiento básico para cualquier postulante a un trabajo o para quienes quieran escalar posiciones dentro de sus organizaciones" (p.1).

Las habilidades blandas son hoy en día un tema muy interesante y al mismo tiempo innovador a nivel de universidad, ya que es la más interesada en que sus estudiantes obtengan dentro de su preparación educativa el mejor perfil que los destaque en las áreas, no solo a nivel profesional, si no también laboral. Según Ferrera, Pérez y Rodríguez (2017), "Dado que la adquisición de competencias es un proceso dinámico, la importancia de las competencias no cognitivas y su efecto sobre los resultados curriculares se incrementa de forma acumulativa en el tiempo" (p. 40).
Para encausar la investigación lo primero que se tiene que tener claro que "Las denominadas habilidades blandas son aquellos atributos o características de una persona, que le permiten interactuar con otras de manera efectiva, lo que generalmente se enfoca al trabajo, a ciertos aspectos de este, o incluso a la vida diaria" (Leiva, 2015, p. 1). Se puede observar que este concepto hace referencia a aptitudes o actitudes que cada individuo debe poseer y como estas influyen en su relación personal.

La importancia del estudio da un panorama general y actual de la situación de las habilidades blandas en los estudiantes de la Universidad Católica de El Salvador, (UNICAES) ${ }^{1}$, y que toda la comunidad universitaria puede hacer uso de los hallazgos obtenidos, como antecedentes para futuros estudios.

\section{Metodología}

La investigación de tipo cualitativa fue orientada a clasificar las estrategias que los docentes utilizan para desarrollar las habilidades blandas en los estudiantes e identificar si las poseen o se les forman en la UNICAES. La población (N) se obtuvo del total de estudiantes inscritos en el ciclo I-2019 de las cuatro facultades que conforman la UNICAES, sede Santa Ana. El número de la muestra se obtuvo mediante la aplicación de la fórmula de población finita, dando como resultado 343 estudiantes.

1. Dentro del documento, la autora hará referencia a este término mediante su acrónimo. 
Para distribuir el número de la muestra entre las diferentes facultades de la UNICAES, se aplicó un muestreo no probabilístico por cuotas, con el propósito de realizar una distribución proporcional a la población (ver tabla 1).

Los resultados se ordenaron para su análisis e interpretación, determinando las habilidades blandas que exige el siglo XXI, cuáles de estas los estudiantes poseen, y la manera cómo se están formando dentro del proceso enseñanza aprendizaje.

Para la recopilación de información se utilizaron diferentes técnicas. Una encuesta, la cual se aplicó a estudiantes que fueron seleccionados mediante la fórmula poblacional que nos arrojó una muestra determinada de estudiantes, dicha cantidad fue distribuida en dos años de primer y quinto año de las diferentes carreras y de las cuatro facultades correspondientes, a través de un cuestionario, el que incluyó diez preguntas cerradas referidas a las habilidades blandas y una pregunta abierta para que los alumnos expresaran el fomento de las mismas en las asignaturas.

Además, se realizó una entrevista estructurada a 24 docentes de la UNICAES, los que fueron seleccionados bajo el criterio de contar con cinco años o más de experiencia como docente en la universidad; dicha cantidad se desglosa de la siguiente manera: dos humanísticas, dos generales, dos de especialidad que en suma son seis docentes que se tomaron por cada una de las cuatro facultades con las que cuenta la Universidad.

Tabla 1. Muestreo no probabilístico por cuotas

\begin{tabular}{|c|c|c|c|c|c|}
\hline Facultad & $\begin{array}{c}\text { Población } \\
\text { estudiantil }\end{array}$ & Porcentaje & $\begin{array}{c}\text { Muestra de } \\
\text { estudiantes }\end{array}$ & $\begin{array}{c}\text { Número de } \\
\text { carreras }\end{array}$ & $\begin{array}{c}\text { Número de } \\
\text { alumnos a } \\
\text { encuestar }\end{array}$ \\
\hline $\begin{array}{c}\text { Ciencias y } \\
\text { Humanidades }\end{array}$ & 2044 & $32 \%$ & 109 & 11 & 10 \\
\hline $\begin{array}{c}\text { Ciencias de la } \\
\text { Salud }\end{array}$ & 1048 & $16 \%$ & 55 & 3 & 18 \\
\hline $\begin{array}{c}\text { Ingeniería y } \\
\text { Arquitectura }\end{array}$ & 1900 & $30 \%$ & 103 & 8 & 15 \\
\hline $\begin{array}{c}\text { Ciencias } \\
\text { Empresariales }\end{array}$ & 1410 & $22 \%$ & 76 & 5 & 56 \\
\hline TOTAL & 6,402 & $100 \%$ & 343 & 27 & \\
\hline
\end{tabular}


Finalmente se aplicó la técnica de la observación en dos clases de cada asignatura abordadas (ver figura 1), para desarrollarla se utilizó una lista de cotejo para verificación de las habilidades blandas de los estudiantes dentro de las aulas.

\section{Resultados}

Inicialmente es necesario establecer que las habilidades blandas, pueden llamarse también, habilidades socioemocionales, inteligencia emocional, habilidades para la vida, mismas que fueron surgiendo en la etapa del marco teórico y que son utilizadas para representar los resultados.

\section{Sobre habilidades blandas que exige el siglo XXI}

Para Vásquez (2018), “Las organizaciones buscan integrar en sus equipos de trabajo talento con excelentes capacidades en el área de formación. Pero también buscan incorporar personal con habilidades no disciplinares que son requeridas en un contexto global con altos estándares que puedan responder a los nuevos retos y entornos cambiantes" (p.1). Las habilidades personales siempre han determinado la función que se desempeñará en la sociedad, así se tiene, que aquel individuo con mayor capacidad para tomar decisiones ocupaba cargos de dirección de los pueblos, como rey o ministro, otros con capacidades para la oratoria, eran exponentes literarios, etc. Pero la sociedad ha ido avanzando y por un momento se centró la atención únicamente en habilidades intelectua- les, reduciendo al hombre solo a este aspecto, cuando realmente es un ser complejo, teniendo sentimientos, emociones, que si están mal encausadas pueden hacer que el intelecto se vea opacado. Por esta situación, la inteligencia emocional se abre camino para mejorar y ayudar a establecer buenas relaciones interpersonales, familiares y sociales. En épocas pasadas bastaba con tener capacidad de cómo tomar una lanza, o cazar un animal; pero ahora, se necesitan muchas capacidades para ser una persona productiva para la sociedad.

Para la interpretación y análisis de los resultados de las encuestas se elaboró una base de datos, luego se realizó un total por pregunta de cada habilidad blanda que fue planteada en la encuesta a los 343 estudiantes, posteriormente se procedió a la representación gráfica ${ }^{2}$ por porcentajes de mayor a menor.

Con respecto a las habilidades blandas que exige el mercado laboral, el 89\% de los estudiantes encuestados de la UNICAES consideraban que el siglo XXI exige la capacidad para trabajar en equipo; el $88 \%$ piensan que la resolución de problemas; el $85 \%$ la adaptación a los cambios y comunicación; el 73\% autodominio y capacidad de articulación; y el 65\% la asertividad (ver figura 2).

Al triangular los resultados de la encuesta, entrevista y observación de clase se logró determinar que las habilidades blandas son com-

1. Los estudiantes, según indicación del instrumento administrado, podían seleccionar más de una opción por pregunta, por eso se hace un análisis porcentual por cada habilidad blanda contemplada en el instrumento. 


\begin{tabular}{|c|c|c|c|}
\hline Facultad & $\begin{array}{c}\text { Materias } \\
\text { Humanísticas }\end{array}$ & Materias generales & $\begin{array}{l}\text { Materias de } \\
\text { especialidad }\end{array}$ \\
\hline $\begin{array}{c}\text { Ciencias y } \\
\text { Humanidades }\end{array}$ & $\begin{array}{l}\text { Desarrollo personal } \\
\text { Teología I } \\
\text { Filosofía }\end{array}$ & $\begin{array}{l}\text { Lectura y composición } \\
\text { Técnicas de redacción } \\
\text { Introducción a la } \\
\text { economía } \\
\text { Aprendizaje de la } \\
\text { Evaluación } \\
\text { Informática Educativa }\end{array}$ & $\begin{array}{l}\text { Introducción al turismo } \\
\text { Gramática Inglesa I } \\
\text { Sistemas } \\
\text { Organizacionales }\end{array}$ \\
\hline Ciencias de la Salud & $\begin{array}{c}\text { Filosofía } \\
\text { Ética } \\
\text { Teología I }\end{array}$ & $\begin{array}{l}\text { Biología } \\
\text { Química }\end{array}$ & Parasitología \\
\hline $\begin{array}{l}\text { Ingeniería y } \\
\text { Arquitectura }\end{array}$ & $\begin{array}{c}\text { Filosofía } \\
\text { Teología I } \\
\text { Desarrollo Personal }\end{array}$ & $\begin{array}{c}\text { Matemática I } \\
\text { Física I }\end{array}$ & $\begin{array}{c}\text { Diseño Arquitectura II } \\
\text { Organización y métodos } \\
\text { Química General }\end{array}$ \\
\hline Ciencias Empresariales & $\begin{array}{c}\text { Filosofía } \\
\text { Teología I } \\
\text { Desarrollo Personal }\end{array}$ & $\begin{array}{l}\text { Matemática I } \\
\text { Introducción a la } \\
\text { Economía }\end{array}$ & Contabilidad de costos I \\
\hline
\end{tabular}

Figura 1. Distribución de asignaturas para la observación. 

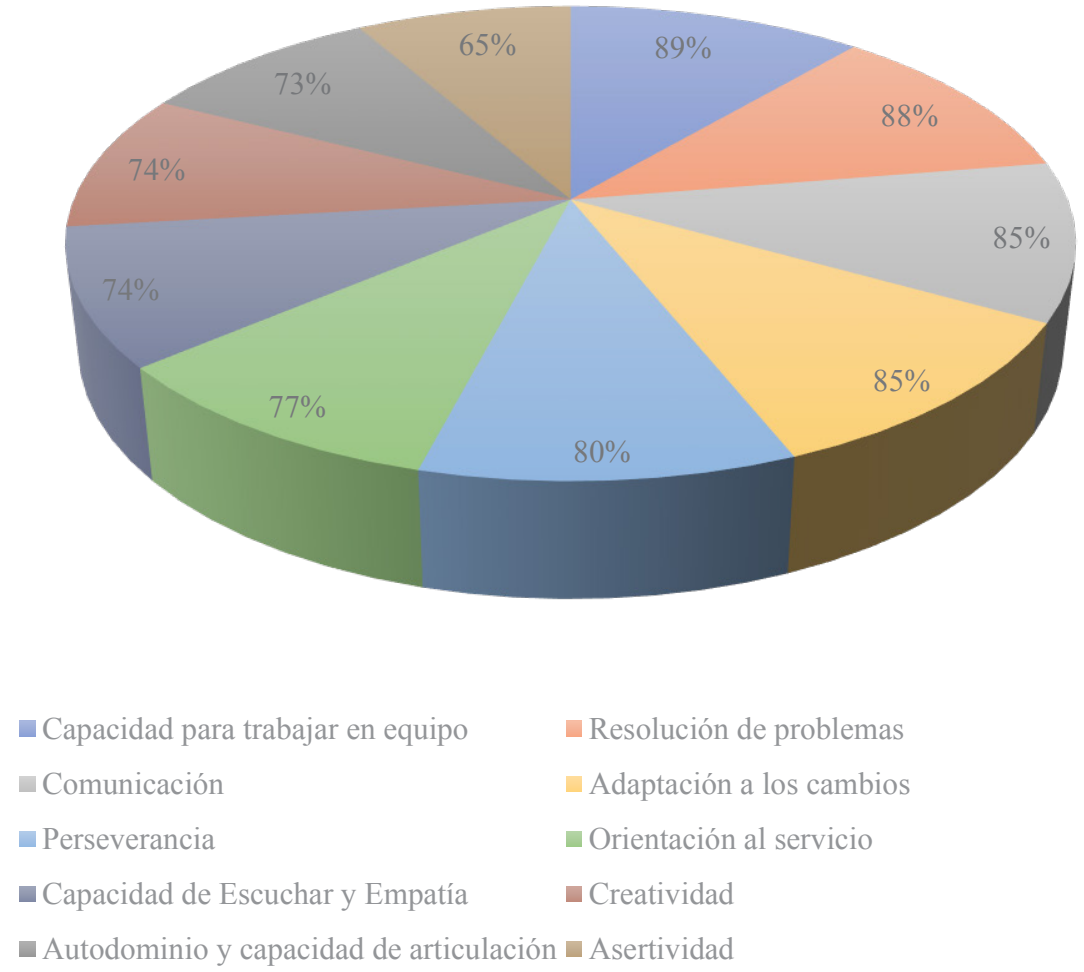

Figura 2. Habilidades blandas que exige el mercado laboral.

petencias o facultades que posee una persona para un mejor desenvolvimiento en su entorno social y estas no son teóricas, más bien se ven reflejadas en la práctica de valores que permiten una formación integral de los mismos.

\section{Habilidades blandas que poseen los estudiantes}

En cuanto a las habilidades blandas que poseen los estudiantes los resultados reflejaron que el $81 \%$ de los estudiantes consideran que poseen la capacidad de trabajar en equipo, y el 80\% la comunicación. Nótese como disminuye el porcentaje cuando se habla de orientación al servicio (48\%); y asertividad (31\%) (ver figura 3).

Por otro parte, los puntos de concordancia en las entrevistas a los docentes consideran que hay ciertas habilidades blandas que necesitan fortalecer los estudiantes en el proceso enseñanza aprendizaje, las cuales son participación activa, puntualidad, respeto, responsabilidad, pensamiento crítico, comunicación, empatía, trabajo en equipo. Mientras que otros mencionan que debe reforzarse la capacidad de negociar, perseverancia, optimismo, proactividad, disciplina e inteligencia emocional, buena presentación, relaciones interpersonales.

Al comparar el resultado del cuestionario con la guía de observacion en el salón de clases, se reflejó que la habilidad blanda de la comunicación y capacidad de trabajar en equipo, son las que mayor porcentaje presentaron. Por el 


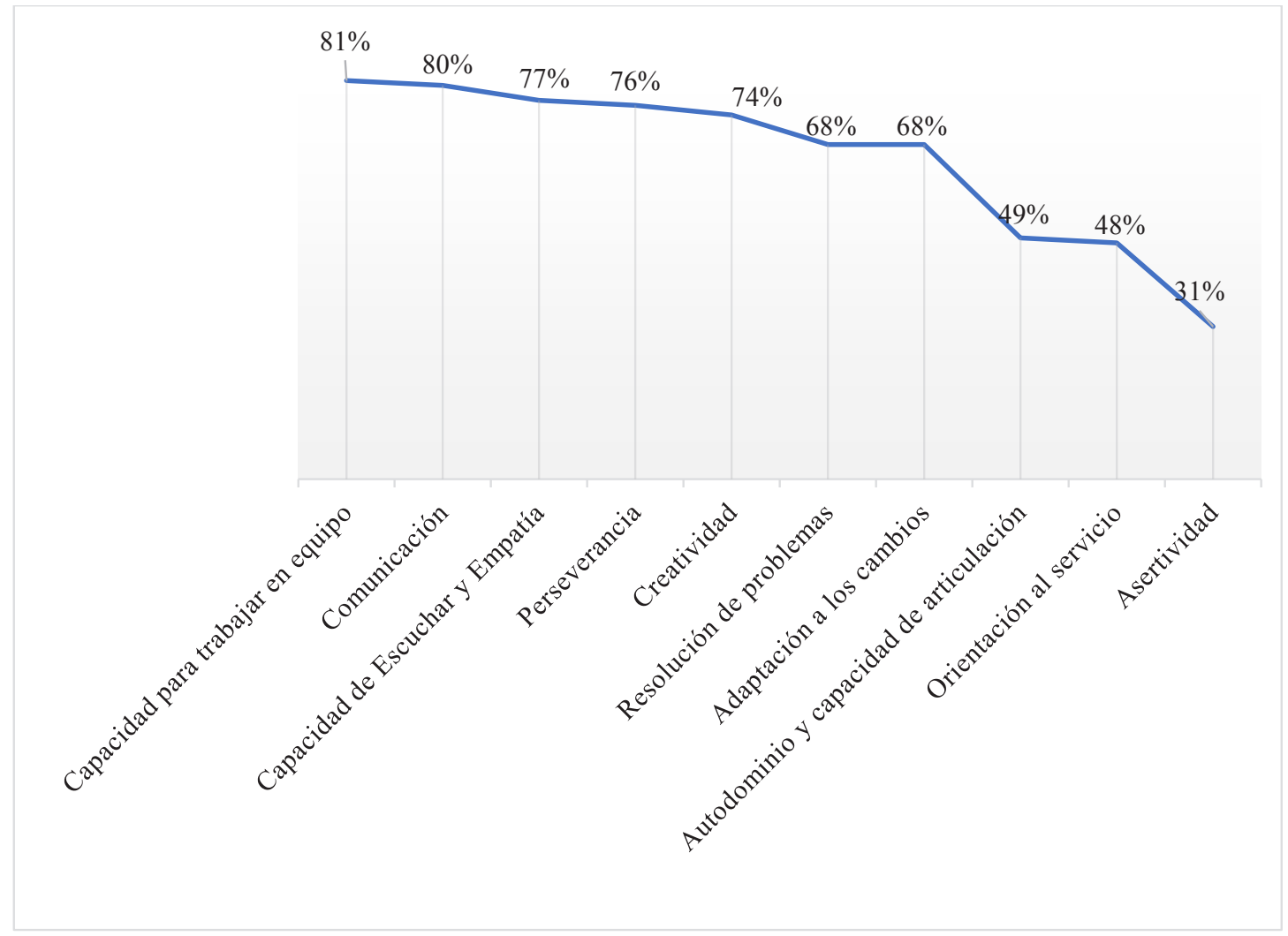

Figura 3. Habilidades blandas que poseen los estudiantes.

contrario, hay una diferencia marcada en la habilidad socioemocional de la creatividad, ya que el $74 \%$ de los estudiantes consideran que la poseen, y en la observación esta resultó visible en tan solo un $42 \%$ de los estudiantes (ver figura 4).

Para la observación en el salón de clase, se hizo el uso de una lista de cotejo, que consistió en diez indicadores sobre las habilidades blandas en el proceso enseñanza aprendizaje para medir su fomento o su ausencia. Luego se trasladaron los resultados a una base de datos, presentando en porcentajes los resultados para una mejor comprensión.
Los resultados obtenidos, mediante la técnica de la encuesta a estudiantes, la guía de observación en los salones de clase y la entrevista a docentes, indicaron que las habilidades con mayor frecuencia o porcentaje según el caso, son el trabajo en equipo y la comunicación. (ver figura 5).

\section{Habilidades blandas que forman los docen- tes en los estudiantes}

De acuerdo a los resultados de la encuesta, los estudiantes consideran que la asertividad es la habilidad blanda con menos fomento por parte del docente. Mientras que la comunicación y la capacidad para trabajar en equipo son las que poseen mayor fomento (ver figura 6). 


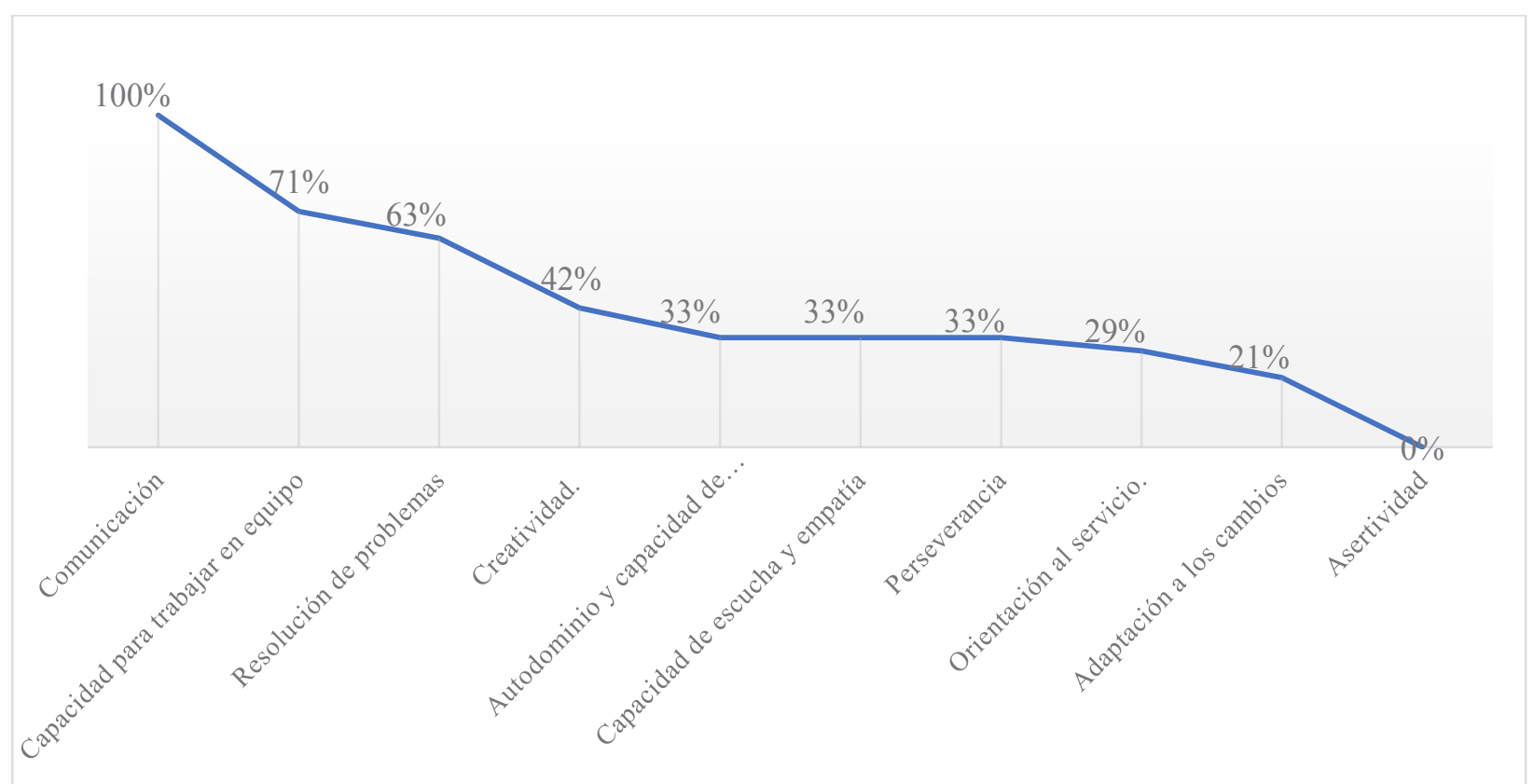

Figura 4. Habilidades que poseen los alumnos observados en el proceso enseñanza aprendizaje.

\begin{tabular}{|c|c|c|}
\hline Encuesta a estudiantes & Observación a clases & Entrevista a docentes \\
\hline $\begin{array}{l}\text { - Capacidad de } \\
\text { escucha } \\
\text { - Comunicación } \\
\text { - Capacidad de } \\
\text { trabajo en } \\
\text { equipo }\end{array}$ & $\begin{array}{ll}\text { - } & \text { Resolución de } \\
\text { problemas } \\
\text { - }\end{array}$ & $\begin{array}{l}\text { - Comunicación } \\
\text { - Empatía } \\
\text { - Trabajo en } \\
\text { equipo }\end{array}$ \\
\hline
\end{tabular}

Figura 5. Matriz de vinculación entre instrumentos. 


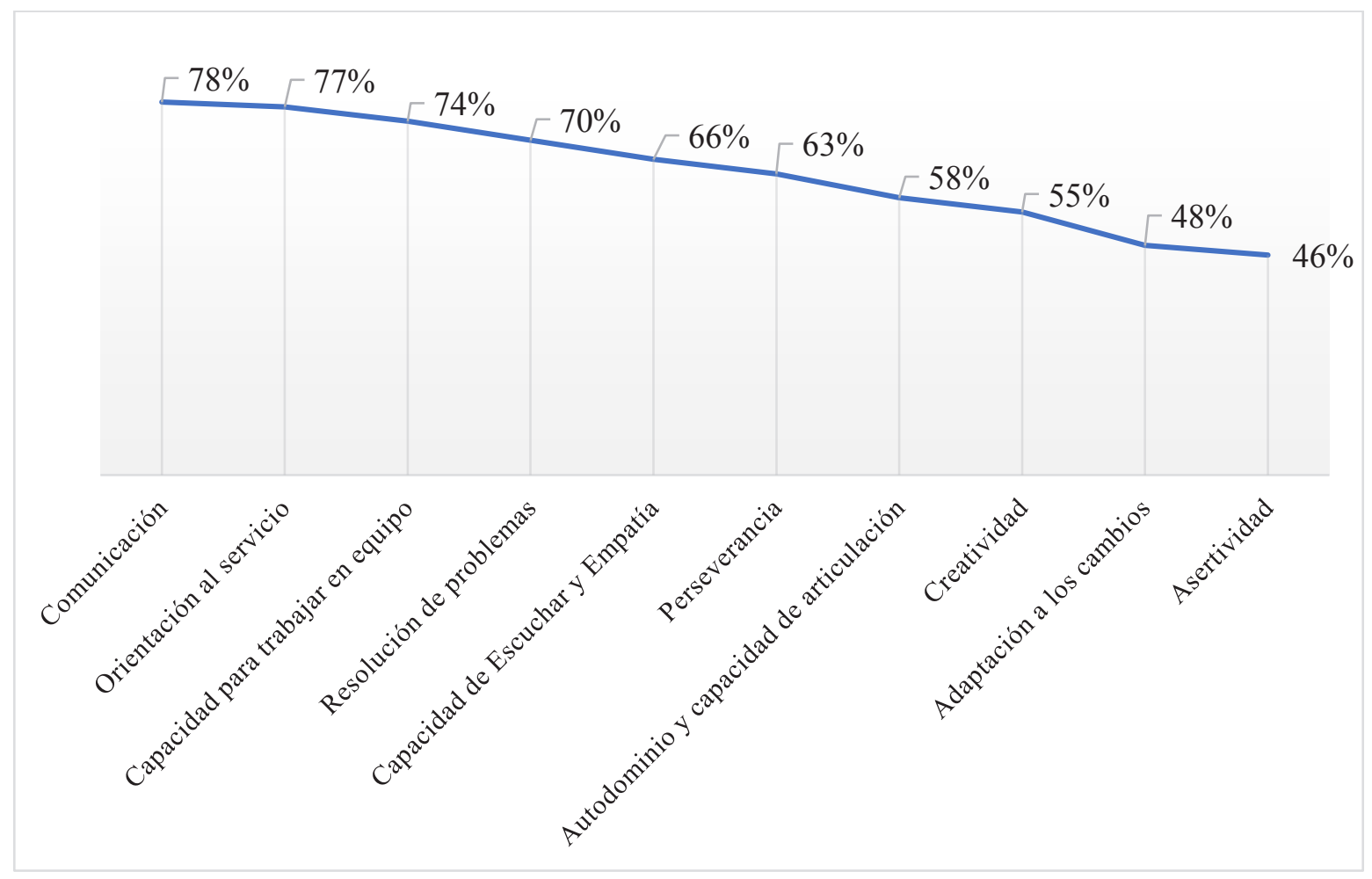

Figura 6. Habilidades blandas que fomenta el docente según la encuesta a estudiantes.

Las habilidades que más sobresalieron en el fomento por parte del docente, según los estudiantes de la UNICAES, son la comunicación, resolución de problemas y orientación al servicio; siendo las dos primeras orientadas a las habilidades socioemocionales internas. La asertividad presentó niveles bajos en los porcentajes, lo cual indica la carencia en su formación o conocimiento.

Los docentes entrevistados coincidieron en que forman y desarrollan en los estudiantes la inteligencia emocional, y no solo el área intelectual, mediante el uso de forma regular de la comunicación, empatía, puntualidad, solidaridad, respeto, amabilidad, trabajo en equipo y responsabilidad (ver figura 7). Sin embargo, otros hacen alusión a nuevas habilidades blandas como la gestión de tiempo, autodisciplina, buena organización, actitud positiva y discusión sobre temas particulares.

Los docentes consultados indicaron que entre las estrategias que utilizan para desarrollar la inteligencia emocional se encuentran las clases magistrales, trabajos individuales, grupales, participación en clases, exposiciones, desarrollo de guías, resolución de proyectos, estudio de casos, resolución de problemas, experiencias personales, transmisión de mensajes positivos, foros, material audiovisual, tareas ex aula, tutorías personales, uso de herramientas digitales, 


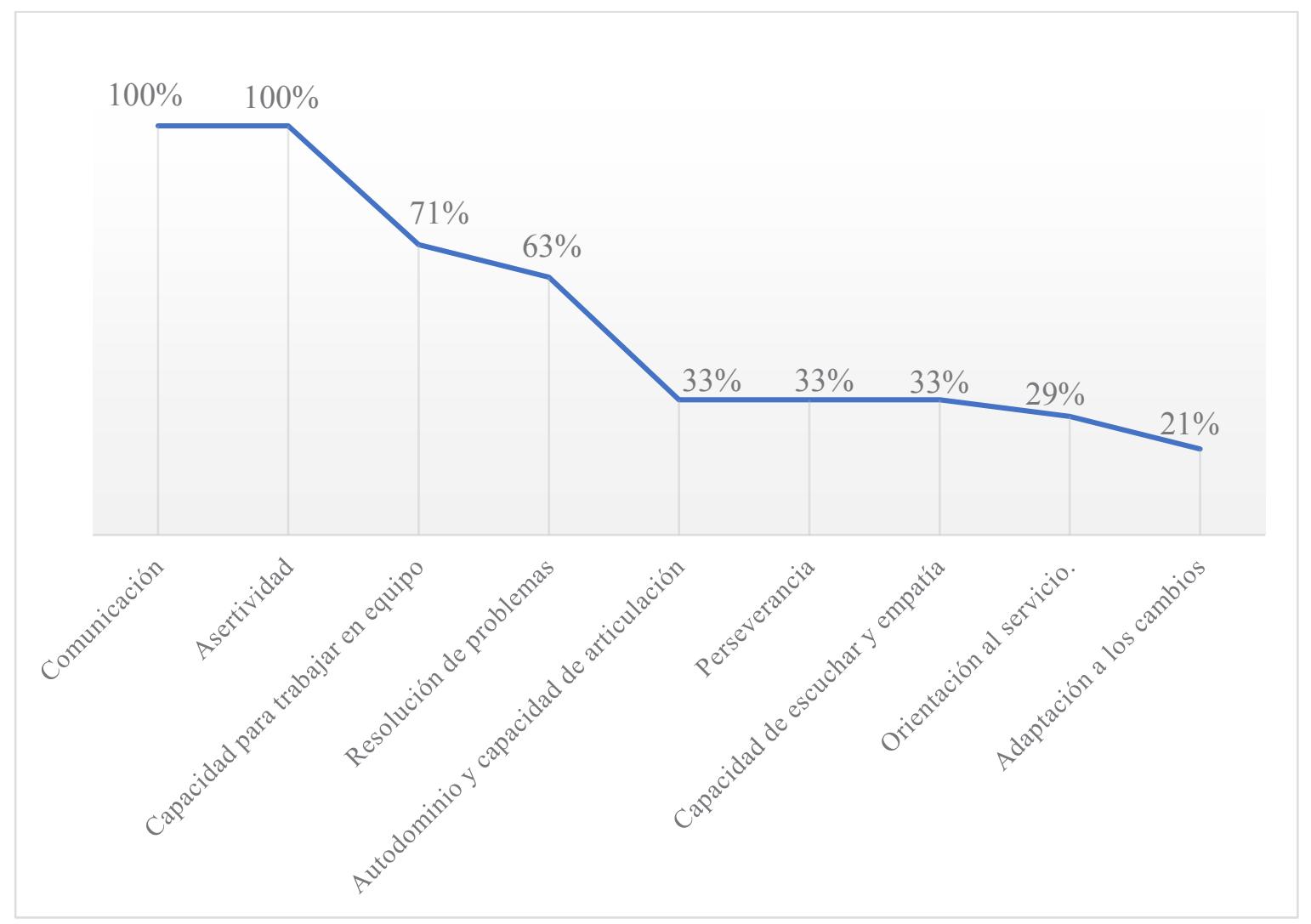

Figura 7. Habilidades blandas que fomenta el docente en el proceso enseñanza aprendizaje.

proyectos e investigaciones; sin embargo, este estudio no tenía como objeto la comprobación de estas técnicas.

Según los resultados obtenidos en la guía de observación que se realizó en los salones de clase, entre las habilidades que obtuvieron mayor porcentaje se encontraron la comunicación y la asertividad; mientras que la adaptación a cambios es la que presenta menores porcentajes.

Otro de los hallazgos obtenidos tiene relación con la capacidad de trabajar en equipo y la comunicación, las cuales son dos de las habilidades que se encuentran en la cima de las que fomentan la UNICAES y las que practican los estudiantes.
Por otro parte, en la resolución de problemas de igual manera hay un porcentaje casi idéntico y por último en la orientación al servicio hay una notable brecha de separación en el sentido que la universidad las fomenta, sin embargo, los alumnos no las practican (ver figura 8).

\section{Discusión}

\section{Las habilidades blandas que exige el siglo XXI}

Para Mott (2018), "Cuando hablamos de habilidades del siglo XXI nos referimos a un amplio conjunto de conocimientos, habilidades, hábitos y rasgos que se estiman críticos para ser exitosos en el mundo actual" (p.14). Esta premisa se confirma en el contexto de la 
UNICAES, ya que el 79\% de los estudiantes en promedio, consideran que ese conjunto de conocimientos y habilidades les exige el mercado laboral salvadoreño. No obstante, en la investigación se hizo un listado cerrado de las habilidades blandas, con esto no se afirma que no existan más habilidades y que estas no las posean los estudiantes.

Es necesario afirmar que en todo trabajo se usan las habilidades, aunque con distinta intensidad, es decir, un carpintero ocupa diferentes habilidades que un abogado, por consiguiente, dependerá de cada autor, país y campo laboral, el uso adecuado de estas. En la actualidad no se pudo encontrar a nivel nacional una investigación que nombrara a las habilidades blandas como tal, solo hay documentación por separada de ellas. Tampoco a nivel institucional sobre la clasificación de la inteligencia emocional, siendo el listado propuesto el respaldado por los alumnos porque todas obtuvieron una respuesta afirmativa en los ítems del cuestionario.

\section{Habilidades blandas que poseen los alumnos}

Según Red (2016), "Por lo tanto, mirar directamente a las habilidades que tienen las personas es una forma más adecuada de aproximar

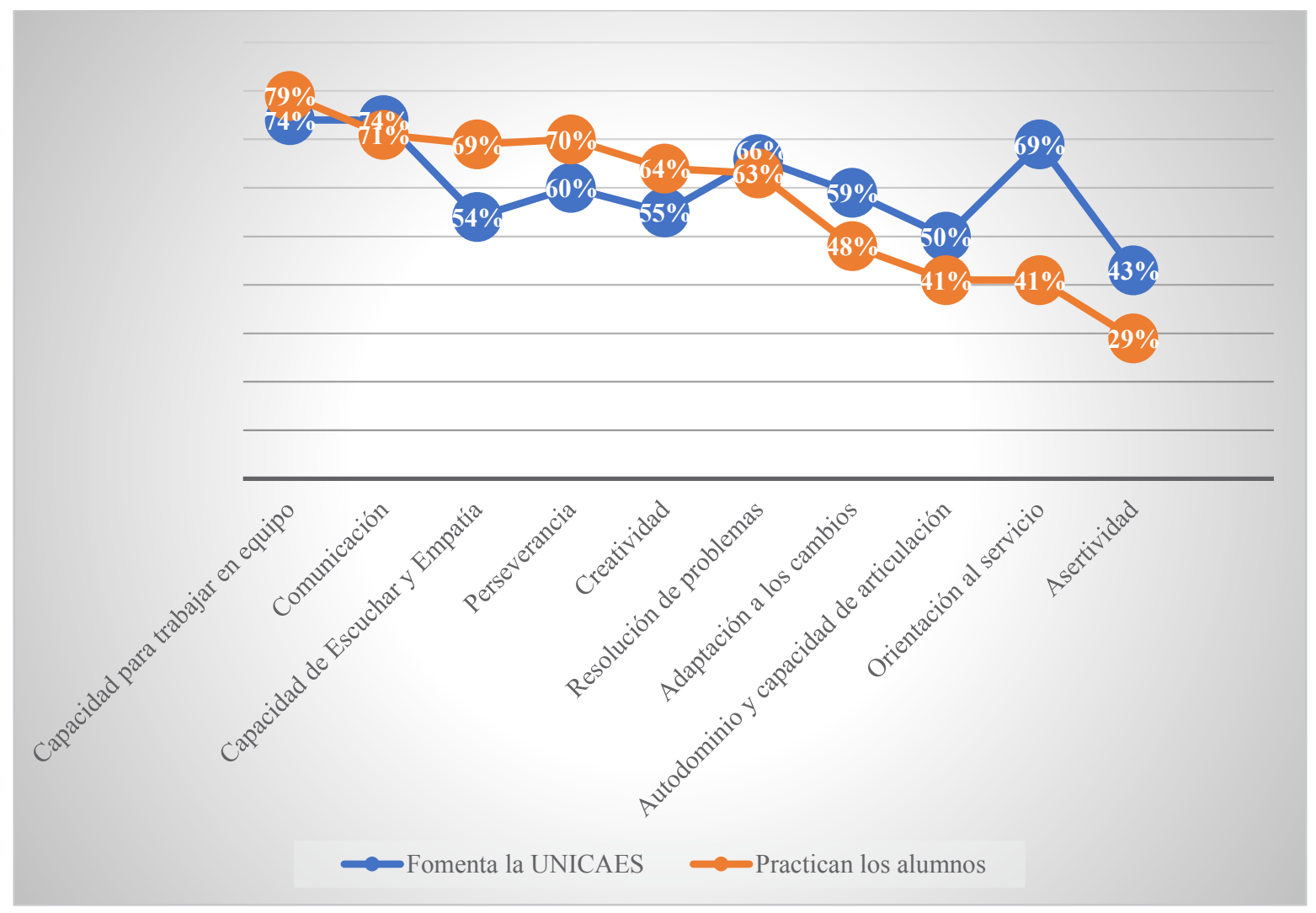

Figura 8. Habilidades blandas que fomenta el docente en el proceso enseñanza aprendizaje. 
su capacidad laboral y el éxito que tendrán en el mercado del trabajo" (p.45). En promedio el $65 \%$ de los estudiantes de la UNICAES consideran que poseen las habilidades blandas, a partir de esto, es necesario establecer altos estándares de desarrollo de habilidades, para que el estudiante pueda fortalecerlas y las logren percibir como parte de sus capacidades, esto le permitirá tener una mejor evaluación laboral.

La habilidad que más llama la atención, por poseer el porcentaje más bajo con un $31 \%$, es la asertividad, ya que se evidenció que no todos los estudiantes la practican y esto se debe en parte al desconocimiento del significado de la palabra. Castanyer (2010) define asertividad, en términos sencillos, como la capacidad para hacer valer los derechos personales. Además, los docentes han afirmado que en el proceso enseñanza aprendizaje es explicada y desarrollada, como ejemplo cuando se les hace saber el reglamento de evaluación. Este hallazgo da pauta para tomar líneas de acción inmediata para salir de esos niveles porcentuales bajos, una alternativa podría ser que el mismo docente vaya haciéndola parte de su vocabulario e incentivando al alumno a practicarla desde los primeros años de formación.

De igual forma, la no participación en clase de forma activa, la nula argumentación sobre las temáticas, la distracción por el ambiente familiar y social se ve reflejado por el poco uso de la asertividad, autodominio, y orientación al servicio, que son tres habilidades que presenta- ron el menor porcentaje en los resultados de la encuesta y en la observación. En este punto, se hace necesario aclarar que el término de habilidades blandas aún sigue siendo un tema desconocido para los docentes y con mayor grado en estudiantes, ya que durante la recolección de los datos se tuvo que definir el concepto a cada uno de los participantes.

Con base en los resultados de la investigación se establece como habilidades prioritarias la comunicación, porque en esencia se es un ser social; el pensamiento crítico, importante porque se necesita tener un criterio sólido sobre las funciones asignadas en el área laboral donde se desempeña y esto va relacionado a tener un buen carácter. ¿En qué organización no surgen problemas?, la respuesta es unánime, en ninguna, por eso la habilidad de manejo de conflictos es necesaria, porque siempre existirán situaciones donde se pondrá a prueba los límites y dominios de la personalidad, y no se está llamado a ser un simple espectador, sino protagonistas de las soluciones.

Se considera que hay habilidades innatas, como la creatividad y la comunicación, dentro de los estudiantes, que las poseen sin mayor esfuerzo. Pero también, se encuentran otras que tienen que ser desarrolladas, fomentadas e inculcadas por los mismos docentes, entre estas se encuentran la participación activa, puntualidad, respeto, responsabilidad, pensamiento crítico, disciplina, buena presentación y capacidad de negociar. 
Cómo están formando los docentes las habilidades blandas

La problemática se aborda desde dos actores principales, a saber: docentes y estudiantes, puesto que son estos los que participan en el proceso enseñanza aprendizaje, teniendo como finalidad "la formación integral de la persona humana", tal como se establece en los fines de la Universidad Católica de El Salvador (Diario Oficial, 2008, p.11).

En las materias numéricas no se puede desarrollar todas las habilidades blandas por la metodología propia de esas asignaturas, puesto que buscan fomentar en el estudiante el desarrollo de análisis e interpretación de datos exactos.

Los docentes utilizan una variedad de estrategias y actividades, que facilitan el desarrollo de las habilidades socioemocionales. Tanto las estrategias y actividades no son lo mismo, cada una ocupa un lugar dentro del proceso enseñanza aprendizaje; las estrategias son: “Acciones planificadas por el docente con el objetivo de que el estudiante logre la construcción del aprendizaje y se alcancen los objetivos planteados", mientras que las actividades son "técnicas y actividades que pueden utilizar para alcanzar los objetivos de aprendizaje". (UNED, 2013, pp. 1-2)

La UNICAES cuenta con tres áreas de estudio agrupadas en: materias humanísticas, materias generales y materias de especialidad. Se pudo constatar que las primeras desarrollan con mayor porcentaje las habilidades blandas, y por impartirse en los primeros ciclos de estudio, se le fomenta al alumno con más énfasis por parte de los docentes.

Una clasificación que se pudo construir a partir de confrontar los resultados y las obras de referencia, son habilidades blandas en internas y sociales, con una perspectiva únicamente pedagógica. Determinando que las habilidades internas son aquellas que en su práctica solo involucra al individuo (creatividad, resolución de problemas, adaptación a los cambios, asertividad, autodominio, capacidad de articulación, perseverancia); y las sociales, se dan cuando para su uso el estudiante entra en relación con las otros (capacidad para trabajar en equipo, comunicación, capacidad de escuchar, empatía, orientación al servicio).

Teniendo en cuenta que ya existe bibliografía acerca de cómo desarrollar las habilidades, sería oportuno adecuar y elaborar un manual propio de la Universidad Católica de El Salvador donde se detalle el tipo de actividad para desarrollar determinada habilidad en clase con los estudiantes; poniendo a disposición del docente una herramienta que le facilite el ejercicio de las mismas dentro del proceso enseñanza aprendizaje.

En relación a lo anterior, es importante demostrar a los estudiantes que en la realidad todo conocimiento es útil; así como, incentivar a descubrir sus capacidades y encausarlas a la proyección social; captar su atención a través de los medios tecnológicos, haciendo uso de debates en línea, foros, entre otros. 
A lo largo de esta investigación surgieron nuevas preguntas que pueden ser parte de futuras investigaciones. A continuación, se plantean cada una de ellas: ¿Cómo mejorar el perfil del estudiante frente a la demanda laboral?, ¿Cómo ayudar al docente a formar las habilidades blandas?, ¿Cómo las habilidades blandas pueden influir en el proceso de ingreso de los estudiantes a nivel superior?, y por último, ¿cómo formar un escenario general de las habilidades blandas a nivel nacional?

\section{Referencias}

Asociación de aprendizaje "Mind the Gap". (2013). Guía metodológica para el desarrollo de las habilidades sociales. https:/psicopedia.org/wp-content/uploads/2013/06/guia_completa-habilidades-sociales-para-adolescentes.pdf

Banco Mundial (2011, 21 de junio). El desarrollo de habilidades socio-emocionales promueve las oportunidades laborales. http://www.bancomundial.org/es/news/feature/2011/06/21/ world-bank-calls-for-prioritizing-the-development-of-cognitive-and-socio-emotional-skills-to-promote-employment-opportunities

Berrios, C. (2017). Manual para técnicos Desarrollo de competencias blandas. https://issuu.com/ carolinabarrios $5 /$ docs/manual_de_competencias_blandas.docx

Brithish Council (2012). Habilidades blandas para el aprendizaje, el trabajo y la sociedad. https://www.britishcouncil.pe/sites/default/files/habilidades_blandas_spanish_core_skills_ brochure_final.pdf

Castanyer, O. (2010). La asertividad, expresión de una sana autoestima. https://www.academia. edu/6893823/LA_ASERTIVIDAD_EXPRESION_DE_UNA_SANA_AUTOESTIMA

Cordero, J, Perez, M y Rodríguez, R. (2017). La relación entre habilidades cognitivas y no cognitivas. Revista de Educación (375), 36-60. https://www.educacionyfp.gob.es/dam/ jcr:3c848cc6-784c-49b8-8139-fe89b31ee0f8/02cordero-pdf.pdf

Cortez, J. (2018, 28 de mayo) ¿Cuáles son las competencias más demandadas por los empleadores alrededor del mundo? Edu Bits. https://observatorio.itesm.mx/edu-bits-blog/competencias-mas-demandadas

Fiszbein, A., Cosentino, C., y Cumsille,B (2016). El desafio del desarrollo de habilidades en América Latina. https://scioteca.caf.com/handle/123456789/1031

Ibarra, L. (2016, 20 de marzo). Las habilidades blandas deben ser parte de la cultura. Blog de Tecnología. http://blogs.laprensagrafica.com/litoibarra/?p=3813 
Estutos de la Universidad Católica de El Salvador (UNICAES). (2008, 10 de junio). Diario Oficial, 11. https://www.diariooficial.gob.sv/diarios/do-2008/06-junio/10-06-2008.pdf

Naranjo, A. (2019). La importancia de las habilidades blandas para la docencia universitaria en el contexto actual. Revista Pensamiento Académico de la Universidad UNIACC, 2 (1). https:// www.revistapensamientoacademico.cl/index.php/Repeac2/article/download/39/41

Molinar, M. (2018, 2 de abril). La educación que necesitamos ante los retos del futuro. Edu Bits. https://observatorio.itesm.mx/edu-bits-blog/educacion-ante-retos-del-futuro

Montt, L. (2018, 16 de octubre). Las competencias para un mundo en constante cambio: habilidades del siglo XXI [sesión de conferencia]. Ceremonia Celebración Día del Profesor Civil de la Armada de Chile. https://www.cned.cl/sites/default/files/conferencia_armada_16-10-2018. pdf. https://www.cned.cl/sites/default/files/conferencia_armada_16-10-2018.pdf

Mujica, J. (2015, enero) ¿Qué son las habilidades blandas y cómo se aprenden? [presentación de diapositivas]. Educrea. https://educrea.cl/que-son-las-habilidades-blandas-y-como-se-aprenden/

Ramírez, J. (2016) ¿Qué son las habilidades blandas y por qué son necesarias desarrollarlas? https://www.universidadescr.com/blog/que-son-las-habilidades-blandas-y-por-que-es-necesario-desarrollarlas/

Rubilar, C. (2016). El desafío de las competencias blandas. Observatorio Duoc. https://www. google.com.sv/search?q=desafio + de + las + competencias $+y+$ habilidades + blandas\&oq=desafio + de + las + competencias $+y+$ habilidades + blandas\&aqs $=$ chrome..69i57.19503j0j7\&sourcei$\mathrm{d}=$ chrome \&ie $=\mathrm{UTF}-8$

Jaramillo, A., Pinzón, C. y Riveros, E. (2019). Programa para el fortalecimiento de habilidades blandas en los docentes del Colegio Las Américas. https://repository.ucc.edu.co/bitstream/20.500.12494/14094/1/2019_habilidades_blandas_docentes.pdf

Singer, M., Guzmán, R., y Donoso, P. (2019). Entrenando competencias blandas en jóvenes. http:/www.inacap.cl/tportal/portales/tp90b5f9d07o144/uploadImg/File/PDF/Entrenando_ Competencias_Blandas_en_Jovenes.pdf

Universia. (2020, 28 de abril). Habilidades blandas: qué son y por qué es importante desarrollarlas. Universia. https:/www.universia.net/cl/actualidad/empleo/habilidades-blandas-que-son-que-importante-desarrollarlas-1078831.html

UNED. (2013). ¿Qué son las estrategias didácticas? https://www.uned.ac.cr/academica/images/ ceced/docs/Estaticos/contenidos_curso_2013.pdf 
Universidad Peruana Cayetano Heredia. (s.f). ¿Cuáles son las habilidades del siglo XXI? https:// ougeg.cayetano.edu.pe/empleabilidad/cuales-son-las-habilidades-del-siglo-xxi

Vera, F. (2016). Infusión de habilidades blandas en el currículo de la educación superior: clave para el desarrollo de capital humano avanzado. Revista Akadèmeia, 7(1), 53-73. http://revistas.ugm.cl/index.php/rakad/article/view/137/129

Vallejo, J. (2019). Análisis de las habilidades blandas necesarias para el éxito de una startup [tesis de maestría, Universidad Técnica Federio Santa María]. Repositorio USM. https://repositorio.usm. cl/bitstream/handle/11673/47156/3560900260737UTFSM.pdf?sequence=1\&isAllowed=y 